\title{
TROMBOSE VENOSA PROFUNDA E SUSPEITA DE TROMBOEMBOLISMO PULMONAR: AVALIAÇÃO SIMULTÂNEA POR MEIO DE ANGIOTOMOGRAFIA PULMONAR E VENOTOMOGRAFIA COMBINADAS*
}

\author{
Laura de Moraes Gomes ${ }^{1}$, Edson Marchiori ${ }^{2}$, Rosana Souza Rodrigues ${ }^{3}$
}

Resumo OBJETIVO: Avaliar a ocorrência e a correlação de tromboembolismo pulmonar (TEP) e trombose venosa profunda (TVP) por meio de um protocolo único de angiotomografia computadorizada. MATERIAIS E MÉTODOS: Estudo prospectivo realizado de julho de 2003 a junho de 2004 no Hospital Copa D'Or, Rio de Janeiro, RJ. Foram analisadas 116 angiotomografias de pacientes com suspeita clínica de TEP. Após o estudo do tórax, com um intervalo de três minutos e sem injeção adicional de contraste, foram obtidos cortes do diafragma até os joelhos, a fim de pesquisar TVP. RESULTADOS: De 116 pacientes, $23(19,8 \%)$ cursaram com TEP, 24 com TVP $(20,7 \%), 15(12,9 \%)$ apresentaram tanto TEP quanto TVP e $9(7,8 \%)$ apresentaram TVP na ausência de TEP. Dos 23 casos positivos de TEP, 15 apresentaram concomitantemente TVP $(65,2 \%)$, enquanto em $8(34,8 \%)$ foi visto apenas TEP. Dos 24 casos positivos de TVP, $15(62,5 \%)$ apresentaram TEP associado, enquanto em $9(37,5 \%)$ foi encontrada TVP isolada. CONCLUSÃO: Os resultados demonstraram a forte relação entre TEP e TVP, a importância de pesquisar TVP nos casos com suspeita de TEP e a utilidade do uso combinado da angiotomografia de tórax e da venotomografia como alternativa de único exame de investigação de TEP e TVP simultaneamente.

Unitermos: Tromboembolismo; Trombose venosa profunda; Angiotomografia; Pulmões.

Abstract Deep venous thrombosis with suspected pulmonary embolism: simultaneous evaluation using combined CT venography and pulmonary CT angiography.

OBJECTIVE: To evaluate the occurrence and the correlation between pulmonary embolism (PE) and deep venous thrombosis (DVT) using a single CT angiography protocol. MATERIALS AND METHODS: This was a prospective study performed at Hospital Copa D'Or, Rio de Janeiro, Brazil from July 2003 to June 2004. We analyzed $116 \mathrm{CT}$ angiography examinations of patients with clinical suspicion of PE. After an interval of approximately three minutes, venous phase images from the diaphragm to the knees were acquired without additional contrast injection in order to determine the presence of DVT. RESULTS: From the 116 patients studied, $23(19.8 \%)$ had PE, $24(20.7 \%)$ had DVT, $15(12.9 \%)$ had both PE and DVT and $9(7.8 \%)$ had DVT alone. Among the 23 cases of PE, $15(65.2 \%)$ had concomitant DVT whereas $8(34.8 \%)$ had only PE. Among the 24 cases of DVT, $15(62.5 \%)$ had associated PE and $9(37.5 \%)$ had DVT alone. CONCLUSION: The results showed a strong relationship between PE and DVT, as well as the importance of investigating DVT in cases with suspected PE and the benefits of performing indirect $C T$ venography after pulmonary CT angiography as a single examination alternative method for simultaneous investigation of PE and DVT.

Keywords: Pulmonary embolism; Deep venous thrombosis; Computed tomography angiography; Lungs.

\section{INTRODUÇÃO}

Trombose venosa profunda (TVP) e tromboembolismo pulmonar (TEP) estão

\footnotetext{
* Trabalho realizado no Departamento de Radiologia da Universidade Federal do Rio de Janeiro (UFRJ) e no Serviço de Radiologia do Hospital Copa D'Or, Rio de Janeiro, RJ.

1. Mestranda do Curso de Pós-graduação em Radiologia da UFRJ.

2. Professor Titular de Radiologia da Universidade Federal Fluminense (UFF), Coordenador Adjunto do Curso de Pós-graduação em Radiologia da UFRJ.

3. Médica Radiologista do Hospital Copa D’Or, Pós-graduada em Radiologia pela UFRJ.

Endereço para correspondência: Dra. Laura de Moraes Gomes. Rua Vitor Valpirio, 219. Pelotas, RS, 96020-250. E-mail lauramgomes@terra.com.br

Recebido para publicação em 20/4/2005. Aceito, após revisão, em 25/5/2005.
}

ligados de maneira inseparável como uma progressão do processo de uma doença. A maioria dos êmbolos pulmonares se origina nas veias profundas das extremidades inferiores ou da pelve ${ }^{(\mathbf{1})}$. Se a suspeita de TEP e TVP é confirmada, nenhuma investigação adicional é necessária ${ }^{(\mathbf{1})}$.

Embora a anticoagulação diminua consideravelmente a taxa de mortalidade por doença tromboembólica recorrente nos pacientes devidamente tratados, a anticoagulação por si só produz elevação das taxas de morbidade e mortalidade. Por esse motivo, a terapia com anticoagulantes durante longo prazo deve ser reservada para pacientes com doença tromboembólica comprovada $^{(2)}$.

O TEP é a mais prevenível causa de mortes hospitalares ${ }^{(3)}$. Como não há sinais e sintomas específicos de TEP, o diagnóstico recai sobre os exames de imagem. Até recentemente, os principais métodos de imagem utilizados para diagnóstico de TEP eram a cintilografia ventilatória e perfusional (V/Q), a ultra-sonografia (US) de veias de membro inferior e a angiografia pulmonar. Apesar do desenvolvimento de vários algoritmos diagnósticos, em muitos casos um diagnóstico definitivo não pode ser feito devido às limitações desses testes ${ }^{(4)}$. 
No início dos anos 90, a introdução da tomografia computadorizada (TC) helicoidal modificou dramaticamente a avaliação das artérias pulmonares, tornando possível o acesso a anormalidades endovasculares e a detecção de trombos intraluminais ${ }^{(\mathbf{5})}$.

O diagnóstico de doença tromboembólica pode ser dividido em TVP e TEP. Entretanto, como o tratamento da TVP (anticoagulação em longo prazo) é o mesmo do TEP na maioria dos casos, a demonstração de TVP freqüentemente torna desnecessário o diagnóstico de $\mathrm{TEP}^{(2)}$.

Moser et al. ${ }^{(6)}$ demonstraram que quase $40 \%$ dos pacientes com doença tromboembólica, mas sem sintomas de TEP, apresentavam achados positivos na cintilografia de V/Q e na radiografia de tórax. Outros autores acharam resultados semelhantes ${ }^{(7)}$. Pacientes com TVP comprovada têm TEP assintomático em $25 \%$ a $50 \%$ das vezes ${ }^{(\mathbf{8 , 9})}$.

Ao se avaliar um paciente com risco de doença tromboembólica, a questão mais importante pode não ser o diagnóstico isolado de TEP. Deve-se tentar também definir quem tem chance de sofrer um novo (e talvez fatal) episódio ${ }^{(9)}$. Um único episódio de TEP hemodinamicamente estável, porém não evidenciado por métodos não invasivos, pode ter menos significado clínico que o desfecho de um grande (e não investigado) trombo em veia profunda que ainda não tenha embolizado ${ }^{(\mathbf{1 0})}$.

Como se acredita que o principal fator de risco para o TEP recorrente seja a TVP residual nas veias proximais das pernas, uma única avaliação tomográfica do sistema venoso profundo e do tórax seria desejável e custo-efetiva. Serviria como uma linha de base para seguimento e poderia guiar intervenções, especialmente colocação de filtro de veia cava inferior ${ }^{(11)}$.

Estudos mostram um aumento no diagnóstico de doença tromboembólica com o uso combinado da venografia por TC nos casos negativos de embolia pulmonar. Em um desses estudos, 91 pacientes tiveram diagnóstico de embolia pulmonar, e um número adicional de 16 pacientes apresentou TVP sem evidência de trombos pulmonares, recebendo o tratamento adequado. Isso representou um aumento de $18 \%$ na porcentagem dos casos positivos ${ }^{(12)}$. Em outro estudo, 31 (5\%) pacientes estudados tiveram TVP detectada na ausência de TEP.
Isso aumentou o número de casos positivos em $36 \%$ (de 85 para 116$)^{(\mathbf{1 3})}$.

Um recente artigo de revisão sugere que a combinação de angiografia pulmonar e venografia por TC (APVTC) constitui o protocolo padrão recomendado para a investigação de TEP, quando se opta por TC como método de imagem. Isso significa que, se um paciente é encaminhado à TC para avaliação de TEP, deve-se estudar concomitantemente o sistema venoso profundo. Não se enquadram nesse protocolo pacientes que já têm estudo recente do sistema venoso por US previamente à suspeita clínica de acometimento pulmonar ${ }^{(\mathbf{1})}$.

Múltiplas investigações validaram a alta acurácia da venografia por TC comparada à US de membros inferiores e mostraram as vantagens de um estudo de imagem não invasivo avaliando simultaneamente TEP e TVP. Apenas alguns minutos a mais são requeridos, e a dose adicional de radiação e o número de imagens podem ser reduzidos utilizando-se aquisições com alguns centímetros de intervalo nos cortes da fase da venografia por TC. Em contraste com a US de membros inferiores, a APVTC permite examinar as veias profundas do abdome, pelve e panturrilha; diagnósticos adicionais ou alternativos podem ser sugeridos ou estabelecidos. A combinação de APVTC representa uma linha de diagnóstico em etapa única no exame imaginológico do TEP, serve como linha de base para o seguimento do paciente e pode ser utilizada para planejar a terapia ${ }^{(11)}$.

O objetivo do trabalho foi avaliar a importância de um protocolo combinado de TC (angiotomografia arterial pulmonar e venotomografia de abdome e membros inferiores) no estudo de pacientes com suspeita de TEP e sua correlação com TVP.

\section{MATERIAIS E MÉTODOS}

Este trabalho foi um estudo prospectivo de pacientes com suspeita de TEP, no período de julho de 2003 a junho de 2004.

Foram incluídos no estudo 116 pacientes consecutivos que realizaram angiotomografia computadorizada de tórax (solicitada pelo médico assistente) e que tiveram suspeita clínica de TEP, encaminhados ao Serviço de Radiologia do Hospital Copa D’Or, Rio de Janeiro, RJ.
Todos os exames foram realizados em tomógrafo GE Light Speed, um aparelho multidetector. Antes de começar a angioTC pulmonar, um escanograma do corpo, incluindo o tórax e se estendendo até os joelhos, foi obtido. Todos os pacientes realizaram angiotomografia de tórax com a seguinte técnica: após um "bolus timing" com $20 \mathrm{ml}$, foram injetados $130 \mathrm{ml}$ de meio de contraste venoso em uma veia do braço, com fluxo de $3 \mathrm{ml} / \mathrm{s}$ em bomba injetora, com o "delay" calculado a partir do bolus. Em uma única apnéia as aquisições eram obtidas utilizando-se cortes de 1,25 mm de espessura com 7,5 mm de incremento ("pitch" 6), com 120 kV e 200 mAs.

Após o estudo da vasculatura pulmonar, com intervalo de aproximadamente três minutos, foram obtidos cortes axiais de $5 \mathrm{~mm}$ de espessura com $15 \mathrm{~mm}$ de incremento, sem nenhuma injeção adicional de contraste, do diafragma até o nível das fossas poplíteas, para se estudar o sistema venoso profundo do abdome e membros inferiores, utilizando-se $120 \mathrm{kV}$ e $100 \mathrm{mAs}$.

O critério diagnóstico utilizado, tanto para TEP quanto para TVP, foi a evidência de falha de enchimento intraluminal, parcial ou total.

Foi criado um banco de dados para armazenamento das informações com relação à presença ou ausência de embolia pulmonar e suas características radiológicas, presença ou ausência de TVP e localização, sintoma principal referido pelo paciente (dispnéia, dor torácica, etc.), diagnósticos alternativos encontrados (pneumonia, derrame pleural, etc.). Os exames foram analisados por, pelo menos, dois radiologistas experientes, e os casos discordantes, resolvidos por consenso.

\section{RESULTADOS}

Foram solicitadas 124 angiotomografias de tórax para pesquisa de TEP no período de julho de 2003 a junho de 2004. Destas, oito foram excluídas do estudo, resultando num total de 116 pacientes selecionados, dos quais $61(52,6 \%)$ eram do sexo feminino e $55(47,4)$, do sexo masculino. A média de idade dos pacientes foi de 68,51 anos, variando entre 10 e 97 anos. A mediana foi de 75 anos, com desviopadrão de 17,72 anos. 
Entre os sinais e sintomas que levaram à suspeita clínica de TEP, o mais freqüente foi a dispnéia $(\mathrm{n}=79 ; 68,1 \%)$, seguido de dor torácica $(\mathrm{n}=28 ; 24,1 \%)$ e hipóxia (n $=21 ; 18,1 \%$ ).

Foram encontrados 23 casos de TEP nos 116 pacientes estudados, correspondendo a $19,8 \%$ da amostra. Dos 93 estudos com resultado negativo para TEP, 18 apresentaram algum tipo de artefato $(19,4 \%)$. Todos eles apresentaram artefatos que prejudicaram a avaliação subsegmentar, e em apenas sete a avaliação de nível segmentar foi prejudicada.

Se fossem excluídos esses 18 pacientes, a frequiência de TEP passaria de 19,8\% para $23,5 \%$.

\section{Achados tomográficos no TEP}

O critério utilizado para diagnóstico de TEP foi a visualização da falha de enchimento intraluminal (Figuras 1, 2 e 3).

Dos 23 casos positivos para TEP encontrados, oito não apresentavam alterações além da(s) falha(s) de enchimento vascular. Nos outros 15 casos de TEP, as alterações radiológicas associadas observadas, por ordem decrescente de frequiência, foram o derrame pleural $(73,3 \%$ ) (Figuras 1 e 2), as opacidades em vidro fosco $(40 \%)$ (Figura 4), as consolidações $(33,3 \%)$, as atelectasias $(20 \%)$, o aumento de calibre da artéria pulmonar $(6,7 \%)$ e o derrame pericárdico $(6,7 \%)$.

\section{Diagnósticos alternativos à TEP}

Nos casos em que a suspeita clínica de TEP não foi confirmada, ou seja, nos casos de angio-TC negativa para TEP, a TC foi capaz de sugerir ou esclarecer vários diagnósticos alternativos que justificariam os sintomas e sinais apresentados pelos pacientes.

Em nosso estudo, foram encontrados diagnósticos alternativos em 60,2\% dos 93 casos com resultados negativos para TEP (56 casos).

Os principais diagnósticos alternativos foram: derrame pleural volumoso $(n=29)$, consolidação $(\mathrm{n}=23)$, linfangite carcinomatosa $(n=4)$, derrame pericárdico $(n=4)$,

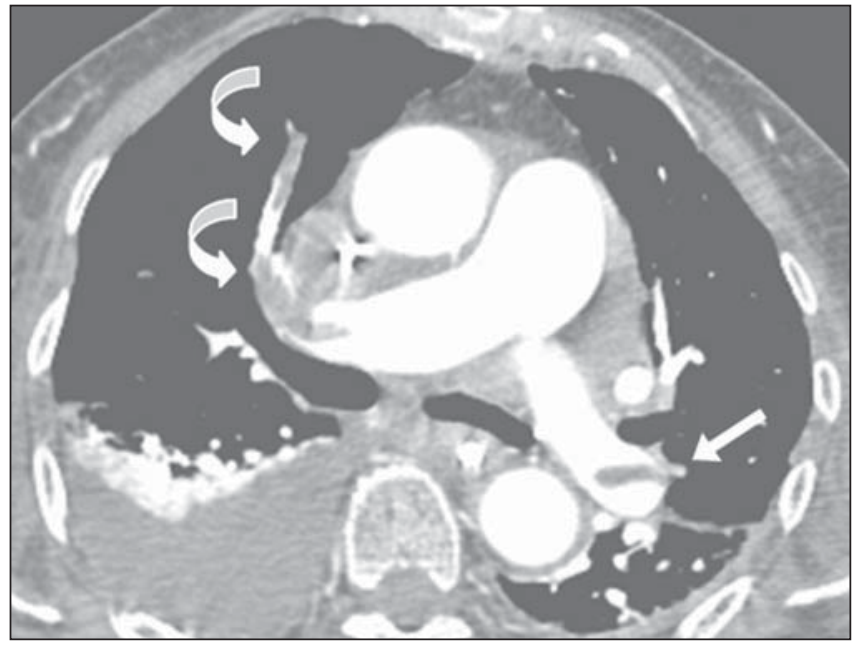

Figura 1. Falhas de enchimento na artéria pulmonar direita (com extensão aos ramos do lobo superior) (setas curvas) e também na artéria para o lobo inferior esquerdo (seta reta).

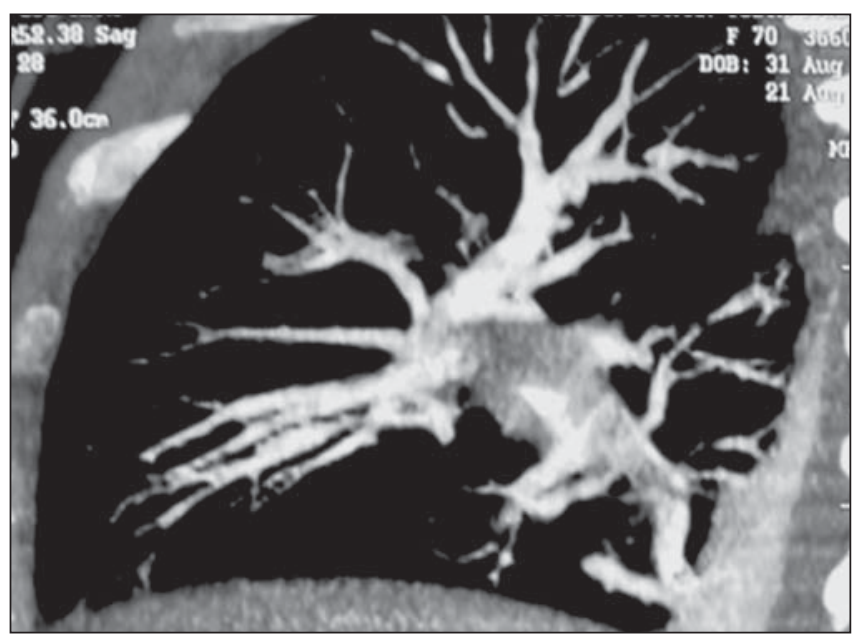

Figura 3. TC espiral em multidetector com reconstrução multiplanar no TEP agudo. Falhas de enchimento na artéria pulmonar esquerda e suas bifurcações.

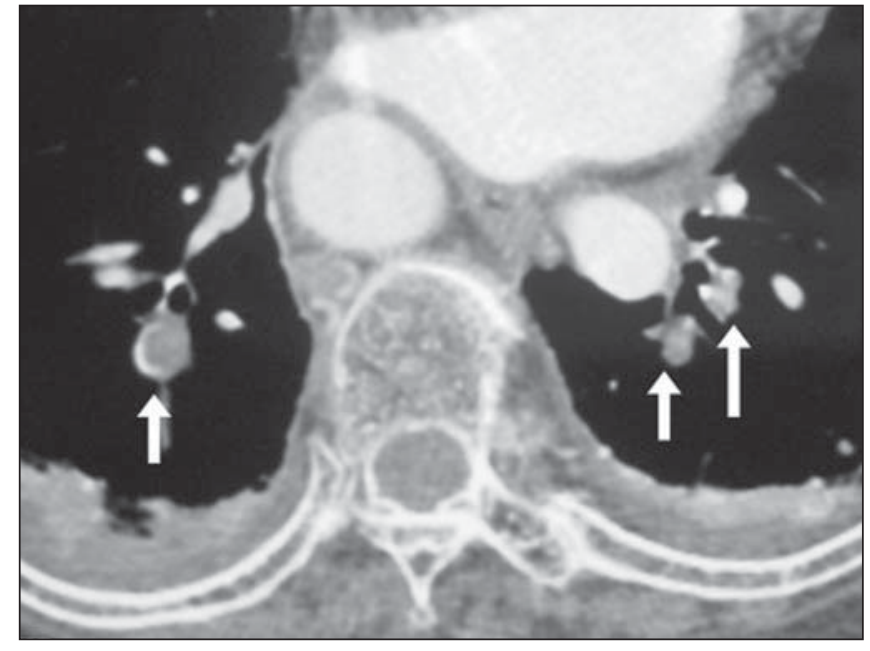

Figura 2. Falhas de enchimento em ramos arteriais segmentares e subsegmentares dos lobos inferiores (setas). Observa-se também pequena efusão pleural bilateral.

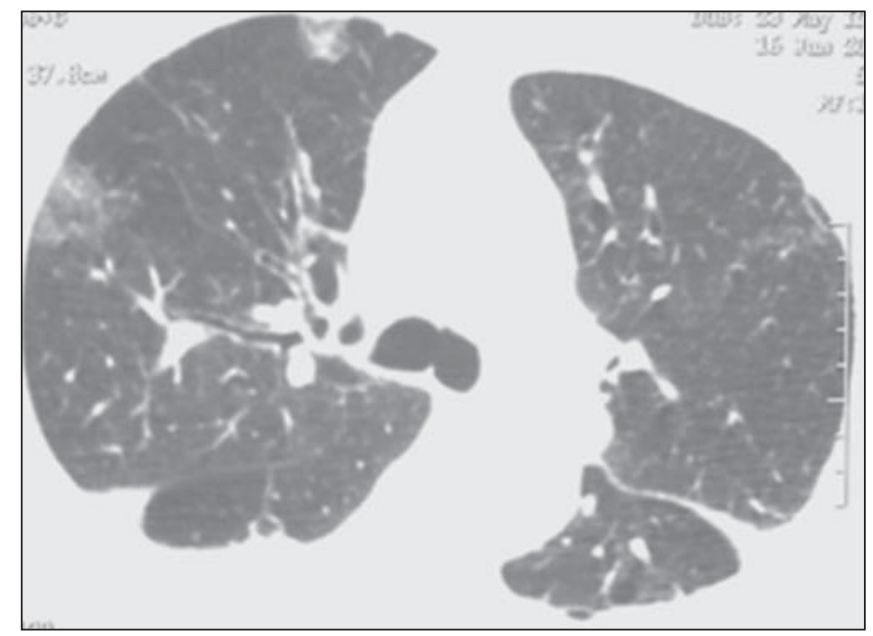

Figura 4. Opacidades em vidro fosco triangulares e periféricas em paciente com TEP agudo (prováveis infartos pulmonares). 
massa mediastinal $(\mathrm{n}=3)$, pneumotórax ( $\mathrm{n}$ $=3)$, bronquiectasias $(\mathrm{n}=1)$ e pneumomediastino $(\mathrm{n}=1)$.

\section{Achados na $\mathrm{TC}$ em relação à TVP}

Nos 116 pacientes estudados houve evidência de TVP em 24 casos, representando $20,7 \%$ da amostra. Destes, nove $(37,5 \%)$ apresentaram somente TVP, ou seja, sem evidência de TEP no exame de tórax.

Entre os 24 casos observados de TVP, a grande maioria se concentrou nas veias femorais e poplíteas (16 e 15 pacientes, respectivamente - Figuras 5, 6 e 7). Também foram evidenciados trombos nas veias ilíacas $(n=9)$ e na veia cava inferior $(n=$ 3 - Figura 8).

Vinte pacientes tiveram trombos nos membros inferiores evidenciados pela TC $(17,2 \%) ; 12$ pacientes apresentaram trombos em veias do abdome (10,3\%).

Dos 24 pacientes com TVP diagnosticada, oito apresentavam trombose simultânea de veias do abdome e dos membros inferiores. Quatro pacientes apresentaram trombose apenas em veias do abdome, o que significa que $33 \%$ dos casos de trombose abdominal foram exclusivamente de abdome, sem associação com trombose de membro inferior.

\section{Sensibilidade e especificidade da TC no diagnóstico de TVP}

Dos pacientes que entraram no estudo, 60 também realizaram US de membros inferiores para pesquisa de TVP, por solicitação do médico assistente.

Os resultados da US de membros inferiores foram então comparados aos da venografia indireta por TC, a fim de avaliar a sensibilidade e a especificidade da TC para o diagnóstico de TVP.

De 15 pacientes com evidência de trombose venosa na US, a TC fez o diagnóstico em 14 casos.

De 45 pacientes com resultado negativo para trombose venosa na US, a TC teve resultado concordante em 44.

Foram encontradas sensibilidade de $93,3 \%$ e especificidade de $97,8 \%$. O teste do qui-quadrado foi utilizado para avaliar o poder estatístico, mostrando que os resultados foram estatisticamente significati$\operatorname{vos}(p<0,001)$.

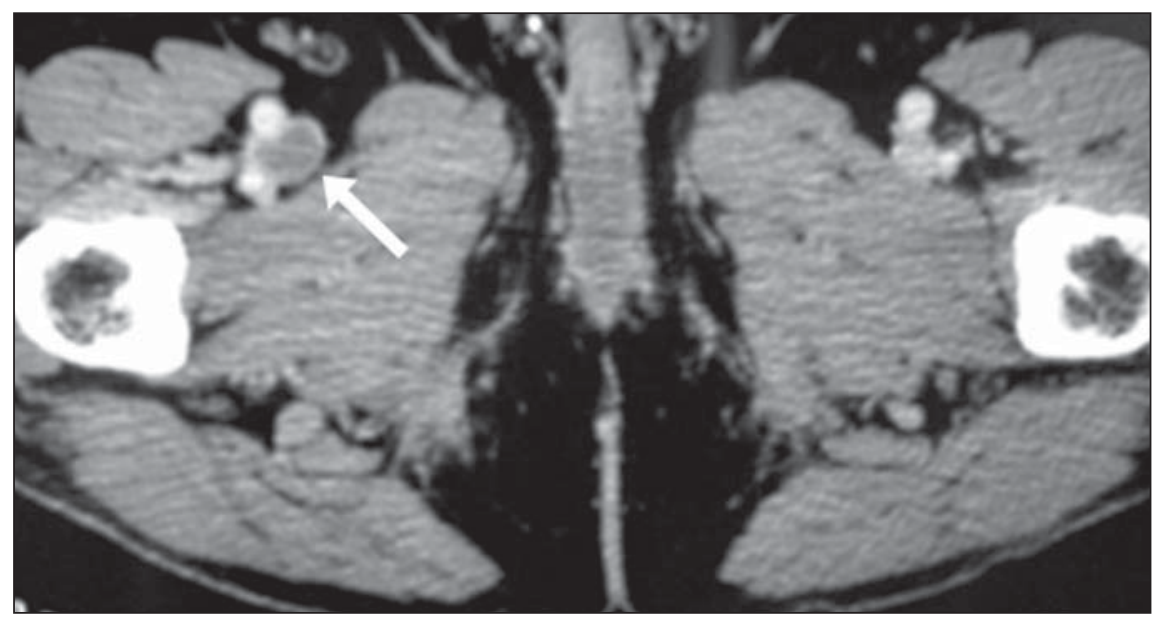

Figura 5. Trombo na veia femoral comum direita (seta).

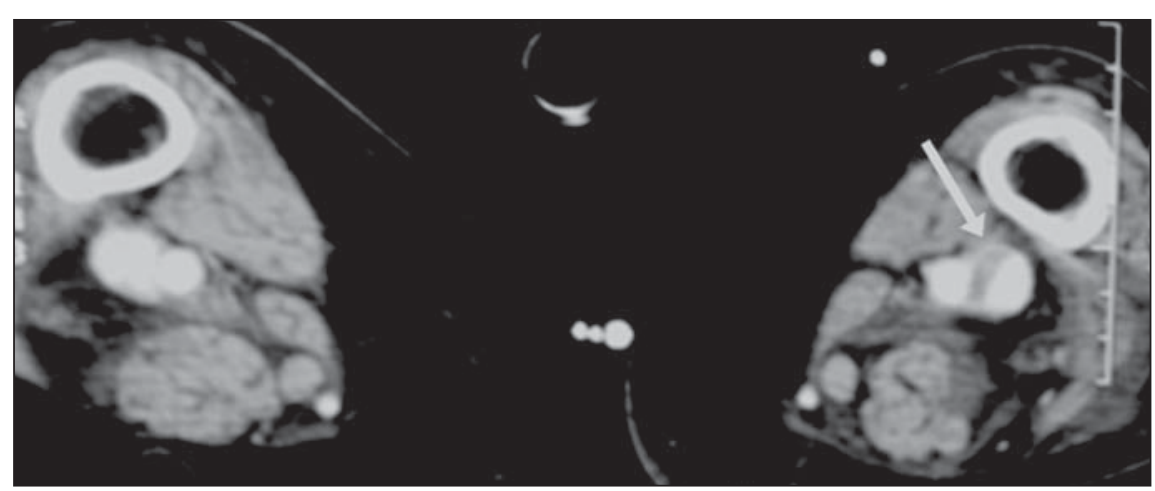

Figura 6. Trombose venosa profunda. Falha de enchimento parcial na poplítea esquerda (seta).

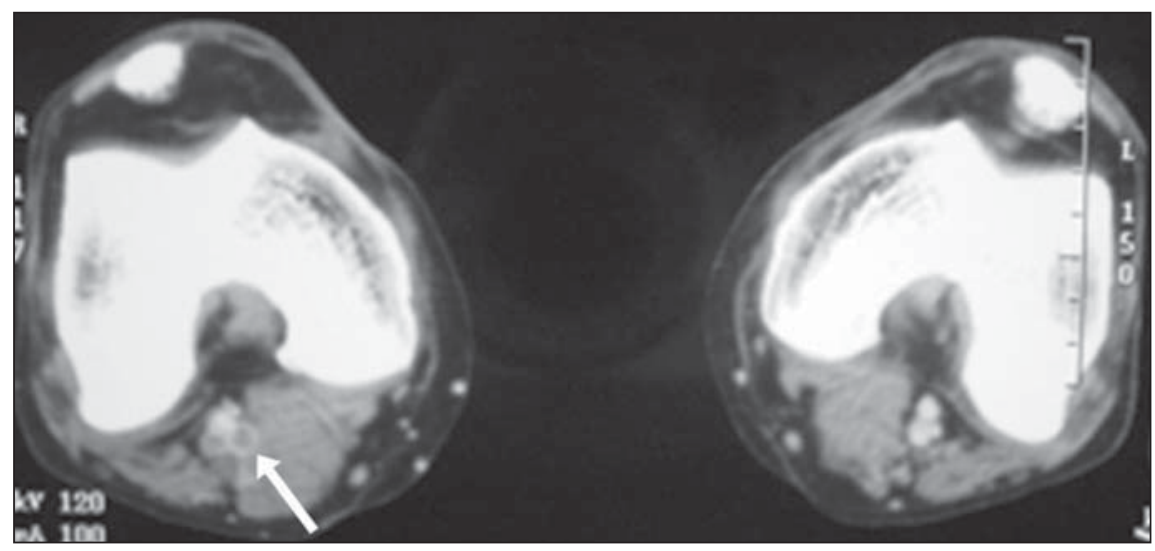

Figura 7. Falhas de enchimento ao nível da fossa poplítea. Observa-se a bifurcação dos trombos para os vasos da panturrilha (seta).

\section{Relação entre TEP e TVP}

De 116 pacientes, $23(19,8 \%)$ cursaram com TEP, 24 com TVP (20,7\%), 15 (12,9\%) apresentaram tanto TEP quanto TVP, 9 $(7,8 \%)$ apresentaram TVP na ausência de TEP e $8(6,9 \%)$ apresentaram TEP na ausência de TVP.
Dos 23 casos positivos de TEP, 15 apresentaram TVP concomitante $(65,2 \%)$, enquanto em $8(34,8 \%)$ foi visto apenas TEP.

Dos 24 casos positivos de TVP, 15 $(62,5 \%)$ apresentaram TEP associado, enquanto em $9(37,5 \%)$ foi encontrada TVP isolada. 
Figura 8. Paciente com angio-TC negativa para TEP apresentando trombo na veia cava inferior (seta).

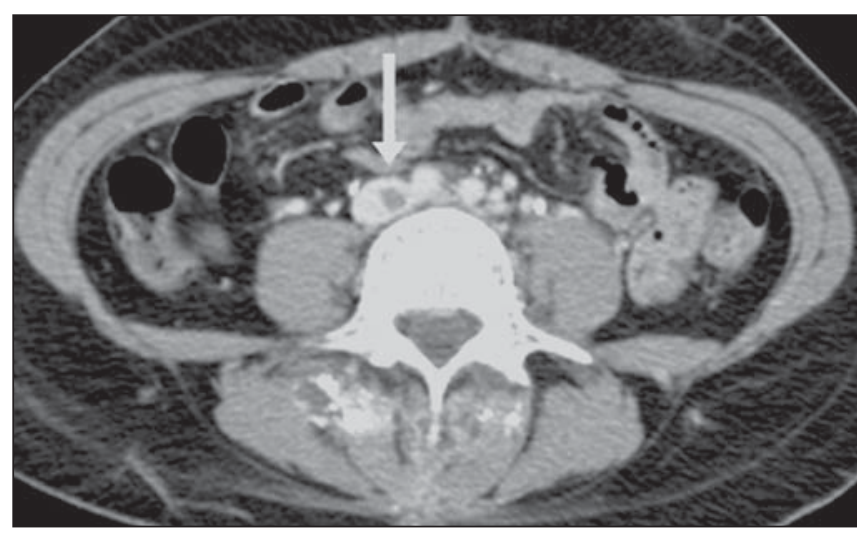

\section{DISCUSSÃO}

\section{Frequiência de TEP}

A freqüência de TEP encontrada em nosso estudo foi de $19,8 \%$. Este resultado se assemelha ao de Cham et al. $^{(\mathbf{1 2})}$, que encontraram $17 \%$.

Entretanto, ao excluírmos os exames que apresentaram algum tipo de artefato, a freqüência subiu para 23,5\%, aproximando-se de outros resultados observados na literatura ${ }^{(\mathbf{1 4})}$. A freqüência de $23,8 \%$ foi encontrada em uma pesquisa que analisou somente pacientes que tinham cintilografia V/Q indeterminada e estudo negativo para $\operatorname{TVP}^{(\mathbf{1 5})}$.

De qualquer maneira, ambos os resultados encontram-se dentro dos padrões da literatura, pois relatos de freqüência de TEP variam de $13 \%$ a $38 \%{ }^{(\mathbf{1 6}-20)}$.

A probabilidade de TEP não foi avaliada em nosso estudo, pois todos os pacientes consecutivos com suspeita de tromboembolia pulmonar foram encaminhados para realizar angiotomografia. Portanto, a proporção de pacientes com baixa probabilidade clínica de TEP que fizeram parte da pesquisa não pôde ser determinada, e sabe-se que, nesse grupo de pacientes, a prevalência de TEP é baixa ${ }^{(\mathbf{2 1})}$.

Como não foi realizado nenhum outro exame para a avaliação do TEP, não foi possível examinar a frequiência de falsopositivos e falso-negativos. O principal motivo pelo qual não se fez um estudo comparativo entre a TC e outro método diagnóstico é que o hospital não dispõe de serviço de medicina nuclear, tornando a comparação da TC com a cintilografia pulmonar ventilatória e perfusional impos- sível. Apesar de a angiografia pulmonar convencional ser disponível em nosso hospital, ela é muito pouco utilizada e não foi realizada em um número de casos suficiente para comparação.

Neste trabalho não foi avaliada a concordância interobservador, mas já é sabido que não há variação com tomógrafos de duas carreiras de detectores ${ }^{(\mathbf{2 2})}$, bem como com os de múltiplas ${ }^{(23)}$.

A idade média (68,5 anos) e a mediana (75 anos) da amostra deixam claro que o TEP é uma doença que ocorre, e portanto é mais investigada, em pacientes idosos. Esse dado é compatível com o da literatura, segundo o qual o risco e a incidência de TEP aumentam acima dos $60 \operatorname{anos}^{(24)}$.

\section{Sinais e sintomas de TEP}

Os principais sinais e sintomas de TEP encontrados em nossa amostra estão de acordo com os descritos na literatura ${ }^{(\mathbf{1 7})}$, com diferenças apenas na ordem de frequiência. Nossos dados foram coletados a partir das justificativas de solicitação de angiotomografia de tórax para investigação de TEP, ou seja, trata-se de dados fornecidos pelo médico assistente.

Como não há relação direta entre o sinal/sintoma e a ocorrência de $\operatorname{TEP}^{(\mathbf{1 7})}$, nosso cálculo foi feito sobre o total da amostra, incluindo tanto os casos positivos quanto os negativos. O resultado reflete, portanto, quais os sinais e sintomas que levaram o médico assistente a investigar a hipótese diagnóstica de TEP.

\section{O papel da TC no diagnóstico do TEP}

A TC "multislice" se mostrou um excelente exame para investigação do TEP, tor- nando possível demonstrar a extensão dos grandes trombos centrais até seus ramos subsegmentares. Isso não só serviu para fazer o diagnóstico de TEP, como também dar uma visão global da doença, além de, simultaneamente, avaliar as condições e comorbidades pulmonares.

Um estudo meta-análise de Moores et $a l .{ }^{(25)}$ concluiu que a taxa de tromboembolismo venoso subseqüente após resultado negativo de angiotomografia pulmonar é similar à encontrada após angiografias convencionais negativas, reforçando a idéia de que é seguro não usar anticoagulação nos pacientes com angio-TC de tórax negativa.

Vários aspectos apontam as utilidades da TC na investigação diagnóstica do TEP. A TC permite avaliação do parênquima pulmonar e estruturas do mediastino concomitantemente com o estudo vascular. Tal vantagem proporcionou a sugestão de outros diagnósticos que não TEP, além de demonstração das alterações existentes nos pacientes com embolia comprovada.

A utilização do protocolo com estudo da fase venosa até o nível dos joelhos estendeu essa vantagem para a obtenção de alterações importantes de abdome e pelve, proporcionando uma idéia global do quadro clínico dos pacientes, fornecendo informações novas ao médico assistente, além de, obviamente, identificar a trombose venosa profunda.

\section{Diagnósticos alternativos}

Uma grande vantagem da TC helicoidal sobre os outros métodos de investigação de embolia pulmonar é a possibilidade de evidenciar diagnósticos alternativos que justificariam a suspeita clínica de TEP.

No presente estudo, em $56(60,2 \%)$ de 93 pacientes sem evidência de TEP, a TC adicionou informações diagnósticas que sugeriram um diagnóstico alternativo. Isso ajudou o médico assistente a compreender melhor o quadro clínico dos pacientes, permitindo que recebessem a terapia adequada para o alívio de seus sintomas. Tal vantagem já havia sido demonstrada por outros autores e foi confirmada nesta pesquisa $^{(15,17)}$.

Em alguns pacientes, os diagnósticos alternativos se sobrepuseram aos achados descritos no TEP, por exemplo, derrame pleural. Nesses casos, foram considerados 
como diagnósticos alternativos aqueles achados que, na ausência de TEP, justificariam os sintomas do paciente (dispnéia, etc.). Além disso, foi feita apenas uma descrição dos achados tomográficos nos casos de TEP, sem estabelecimento de relação causa/conseqüiência.

\section{O uso nos pacientes graves}

Como a TC, principalmente no que diz respeito aos aparelhos "multislice", é um método de exame rápido e não invasivo, torna-se um meio muito útil no diagnóstico dos pacientes graves. Devido às bem conhecidas dificuldades de se pesquisar TEP agudo nos pacientes com doença respiratória subjacente, o benefício da TC "multislice", a qual permite a obtenção de um maior número de exames interpretáveis até o nível do leito arterial pulmonar subsegmentar comparativamente com a TC helicoidal simples, é mais do que evidente ${ }^{(26,27)}$.

Além disso, a presença de doença respiratória subjacente não afeta o valor preditivo negativo da angiotomografia pulmonar (28)

Em nosso trabalho, muitos pacientes se encontravam em estado geral ruim ou grave, sendo vários encaminhados da unidade de terapia intensiva, que necessitavam de um exame rápido e eficaz.

Mesmo nos casos dos pacientes em uso de ventilação mecânica, sonda nasogástrica e outros equipamentos comumente utilizados no paciente grave, o exame ficou de qualidade satisfatória, sendo possível a visualização dos trombos arteriais pulmonares por meio da angiotomografia computadorizada.

\section{Limitações}

Mesmo sendo um bom método de exame, é claro que a TC também possui suas limitações. Remy-Jardin et al. ${ }^{(27)}$ já descreveram, como principais causas de detecção inadequada de ramos segmentares, o realce subótimo, os artefatos de movimento e os efeitos de volume parcial. Já para os ramos subsegmentares, em primeiro lugar ficaram os efeitos de volume parcial, seguidos de realce subótimo e artefatos de movimento.

Exames com artefatos ocorreram em 18 pacientes de nosso estudo, mas em todos eles foi possível fazer a avaliação dos vasos arteriais pulmonares principais e lobares, o que excluía trombos centrais. Em 11 desses pacientes, a avaliação dos ramos segmentares pôde ser feita e o resultado foi considerado negativo para TEP até o nível segmentar. Como a conseqüência do tromboembolismo subsegmentar isolado tem suas controvérsias, esses exames podem ser considerados negativos com certa tranqüilidade, pois mesmo a angiografia pulmonar convencional não é precisa para o diagnóstico de TEP limitado a esses níveis (embolia pulmonar subsegmentar isolada) ${ }^{(29)}$.

Um estudo recente mostrou que pacientes com TEP subsegmentar isolado que não receberam terapia com anticoagulantes não apresentaram episódio recorrente de TEP durante o seu seguimento. Nesse mesmo estudo, a maioria dos pacientes com resultado inconclusivo para TEP na angiotomografia teve anticoagulação suspensa pelo médico assistente, sem nenhum outro exame de imagem adicional para investigação de embolia pulmonar. Não houve registro de efeitos adversos decorrentes dessa decisão clínica ${ }^{(30)}$. O diagnóstico de TEP ficou limitado a excluir trombo central em apenas sete pacientes, que tiveram artefatos em ramos arteriais pulmonares segmentares.

Uma vez que metade da nossa amostra foi composta por pessoas acima de 75 anos, é natural que algumas tivessem reserva pulmonar prejudicada (ou mesmo acometimento de outro sistema orgânico), fazendo com que as condições do exame não fossem tecnicamente perfeitas. Deve-se ressaltar que nem sempre os artefatos prejudicaram o exame como um todo, muitas vezes ficando restrito a um segmento pulmonar. Esse fato, somado ao grau de suspeita clínica de TEP, fez com que o resultado fosse considerado definitivo, sem necessidade de complementação. Pacientes com exames de TC de má qualidade e negativos para TEP, mas com elevada suspeita clínica, devem ser submetidos à angiografia pulmonar ${ }^{(31)}$. O protocolo incluindo cortes tomográficos até os joelhos também foi muito útil nesses casos, pois o afastamento da hipótese de TVP falava a favor de um diagnóstico negativo, enquanto a constatação de um trombo venoso no abdome ou membro inferior tornava o diag- nóstico automaticamente positivo, independente de a visualização de trombos pulmonares ter sido prejudicada ou não.

\section{Diagnóstico de TVP}

O uso da US na pesquisa de TVP dos membros inferiores já está consagrado pelo fato de ser um exame sensível, específico, além de ser um método não invasivo. Ambas as técnicas - compressão e Doppler colorido - , principalmente se utilizadas em conjunto, são úteis no diagnóstico $^{(32-34)}$. Há quase duas décadas, na literatura já se relatava que a venografia pode ser dispensada em casos em que a existência de TVP se confirme com métodos não-invasivos e não se pretenda proceder à desobstrução cirúrgica ${ }^{(35)}$.

A TC começou a aparecer como uma alternativa à US no diagnóstico da TVP depois que seu uso ficou consolidado para o diagnóstico do TEP. Vários autores começaram a pesquisar a utilidade da TC para a avaliação de TVP, pois, uma vez que a tomografia era considerada bom método para visualização de trombos arteriais, também o poderia ser para trombos venosos. $\mathrm{O}$ fato de se poder aproveitar o mesmo contraste utilizado em uma angio-TC de tórax facilitou os estudos e acabou revelando uma grande vantagem da TC. Aproveitando o mesmo contraste utilizado para o estudo do tórax, em apenas alguns minutos a mais de exame é possível pesquisar trombos no sistema venoso do abdome,

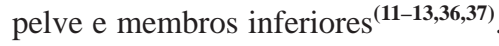

A sensibilidade e a especificidade da venotomografia indireta comparada à US são bastante elevadas. Em um estudo, ambas foram de $100 \%{ }^{(37)}$. O grupo de Loud et al. $^{(13)}$ examinou 650 pacientes, dos quais 58 tinham TEP e TVP e 31 tinham apenas TVP vistos na tomografia. A sensibilidade encontrada pelos autores foi de $97 \%$ e a especificidade, de $100 \%$. Em nosso trabalho, foi encontrada sensibilidade de $93,3 \%$ e especificidade de $97,8 \%$. Esses valores, embora um pouco inferiores aos citados anteriormente, mostram que a venografia indireta por TC é um bom método para o diagnóstico de TVP de membros inferiores. Nossos resultados ficaram mais próximos aos de Coche et al. ${ }^{(36)}$, que encontraram $93 \%$ de sensibilidade e $97 \%$ de especificidade. 
Nosso protocolo de exame seguiu basicamente os modelos descritos na literatura para avaliação simultânea de TEP e TVP pela $\mathrm{TC}^{(\mathbf{1 2 , 1 3 , 3 6 )}}$. A quantidade de contraste $(150 \mathrm{ml})$ e o tempo de intervalo entre o estudo arterial e venoso (entre dois e três minutos) foram quase iguais. No estudo de Coche et al. ${ }^{(\mathbf{3 6})}$, foram utilizados torniquetes nos membros inferiores, que foram mantidos um pouco elevados durante o exame, e a aquisição das imagens começou a partir da panturrilha em direção à pelve. Em nosso estudo, as imagens foram obtidas em decúbito dorsal convencional e em sentido crânio-caudal. O grupo de Cham et al. ${ }^{(\mathbf{1 2})}$ fez cortes das cristas ilíacas até as fossas poplíteas, excluindo as panturrilhas, por considerar não relevante. Foram utilizados cortes de $10 \mathrm{~mm}$ de espessura, o dobro do nosso estudo, que utilizou $5 \mathrm{~mm}$. Loud et al. ${ }^{(13)}$ iniciaram a fase de aquisição venosa da mesma maneira que a nossa, isto é, a partir do diafragma, só que com intervalos de $5 \mathrm{~cm}$. Em dois desses trabalhos ${ }^{(\mathbf{1 2}, \mathbf{1 3})}$, o estudo foi multicêntrico, o que fez com que a técnica variasse um pouco de hospital para outro, pois os tomógrafos eram diferentes. Em nenhum deles foi citado o uso de tomógrafo multidetector como o do nosso estudo.

Como nos trabalhos supracitados, em nossa pesquisa ficou evidente a utilidade da TC para diagnosticar TEP e TVP, bem como demonstrar a forte relação entre as entidades. Dos 23 pacientes com TEP, 15 $(65,2 \%)$ tinham TVP. Nove de 116 pacientes $(9,7 \%)$ tiveram apenas TVP detectada na tomografia, ou seja, foram diagnosticados graças à utilização do protocolo, freqüência superior à de Loud et al. ${ }^{(\mathbf{1 3})}$, em que 31 de 650 pacientes tiveram TVP isolada (5\%), e de Cham et al. ${ }^{(\mathbf{1 8})}$, que encontraram 43 em 1.590 pacientes (3\%). Mesmo assim, com percentuais aparentemente baixos de TVP isolada, esses autores ${ }^{(\mathbf{1 3 , 1 8})}$ ressaltam a importância da realização do estudo de sistema venoso, pois houve um incremento de $36 \%$ e $20 \%$ nos casos positivos, respectivamente. Em nosso estudo, com o acréscimo dos nove casos de TVP sem TEP, o aumento foi de 23 para 32 casos (o que equivale a $39 \%$ dos casos positivos). Uma possível explicação para termos encontrado um percentual um pouco mais elevado de TVP é o fato do nosso protocolo incluir cortes mais finos (5 mm) e intervalos menores (15 mm) nas aquisições da fase venosa. Nos outros estudos foram utilizados cortes de $10 \mathrm{~mm}$ e intervalos até $5 \mathrm{~cm}^{(\mathbf{1 3}, 18)}$.

Apesar de a US ser um exame eficiente nos casos sintomáticos de TVP, não envolver nenhum meio de contraste nem radiação ionizante, e ser portátil, ela apresenta desvantagens em relação à venotomografia: é operador-dependente; tem uso questionável acima do ligamento inguinal e abaixo do joelho; é menos sensível nos casos assintomáticos; pode ter dificuldades nas variantes anatômicas; pode causar desconforto ao paciente; e possui uso limitado nos pacientes obesos ${ }^{(\mathbf{1 1})}$.

Outra vantagem inquestionável do protocolo combinado é a avaliação das veias do abdome e da pelve. Em nosso estudo, houve três casos de trombo em veia cava inferior e nove em veias ilíacas, que poderiam ter ficado sem diagnóstico se a angioTC de tórax fosse complementada apenas com US de membros inferiores. Dos 12 casos de TVP abdominal detectados na TC, quatro $(33,3 \%)$ eram exclusivos do abdome e oito $(66,6 \%)$ também cursavam com trombo de membro inferior.

Por esses motivos, a combinação da venografia e da angiografia pulmonar por TC helicoidal é citada como protocolo padrão de investigação de TEP, exceto quando a US de membros inferiores tiver sido realizada antes de se fazer a $\mathrm{TC}^{(\mathbf{1 1})}$.

\section{REFERÊNCIAS}

1. Wolfe TR, Hartsell SC. Pulmonary embolism: making sense of the diagnostic evaluation. Ann Emerg Med 2001;37:504-514.

2. Johnson MS. Current strategies for the diagnosis of pulmonary embolus. J Vasc Interv Radiol 2002;13: 13-23.

3. Ryu JH, Olson EJ, Pellikka PA. Clinical recognition of pulmonary embolism: problem of unrecognized and asymptomatic cases. Mayo Clin Proc 1998;73: 873-879.

4. Henschke CI, Mateescu I, Yankelevitz DF. Changing practice patterns in the workup of pulmonary embolism. Chest 1995;107:940-945.

5. Remy-Jardin M, Mastora I, Remy J. Pulmonary embolus imaging with multislice CT. Radiol Clin North Am 2003;41:507-519.

6. Moser KM, Fedullo PF, Littejohn JK, Crawford R. Frequent asymptomatic pulmonary embolism in patients with deep venous thrombosis. JAMA 1994; 271:223-225.

7. Meignan M, Rosso J, Gauthier H, et al. Systematic lung scans reveal a high frequency of silent pulmonary embolism in patients with proximal deep venous thrombosis. Arch Intern Med 2000;160: 159-164.

8. Huisman MV, Buller HR, Cate JW, et al. Unexpected high prevalence of silent pulmonary embolism in patients with deep venous thrombosis. Chest 1989;95:498-502.

9. Nielsen HK, Husted SE, Krusell LR, Fasting H, Charles P, Hansen HH. Silent pulmonary embolism in patients with venous thrombosis. Incidence and fate in a randomized, controlled trial of anticoagulation versus no anticoagulation. J Intern Med 1994;235:457-461.

10. Dalen JE. When can treatment be withheld in patients with suspected pulmonary embolism? Arch Intern Med 1993;153:1415-1418.

11. Katz DS, Loud PA, Bruce D, et al. Combined CT venography and pulmonary angiography: a comprehensive review. RadioGraphics 2002;22(Spec No):S3-S19.

12. Cham MD, Yankelevitz DF, Shaham D, et al. Deep venous thrombosis: detection by using indirect $\mathrm{CT}$ venography. The Pulmonary Angiography-Indirect CT Venography Cooperative Group. Radiology 2000;216:744-751.

13. Loud PA, Katz DS, Bruce DA, Klippenstein DL, Grossman ZD. Deep venous thrombosis with suspected pulmonary embolism: detection with combined CT venography and pulmonary angiography. Radiology 2001;219:498-502.

14. Dalen JE. Pulmonary embolism: what have we learned since Virchow? Natural history, pathophysiology, and diagnosis. Chest 2002;122:1440-1456.

15. Ferretti GR, Bosson JL, Buffaz PD, et al. Acute pulmonary embolism: role of helical CT in 164 patients with intermediate probability at ventilationperfusion scintigraphy and normal results at duplex US of the legs. Radiology 1997;205:453-458.

16. Blachere $\mathrm{H}$, Latrabe V, Montaudon $\mathrm{M}$, et al. Pulmonary embolism revealed on helical CT angiography: comparison with ventilation-perfusion radionuclide lung scanning. AJR 2000;174:10411047.

17. Kim KI, Müller NL, Mayo JR. Clinically suspected pulmonary embolism: utility of spiral CT. Radiology 1999;210:693-697.

18. Cham MD, Yankelevitz DF, Henschke CI. Thromboembolic disease detection at indirect CT venography versus CT pulmonary angiography. Radiology 2005;234:591-594.

19. Bok H, Hugo H, Mendes FG, et al. Achados clinicopatológicos na tromboembolia pulmonar: estudo de 24 anos de autópsias. J Bras Pneumol 2004;30: 426-432.

20. Mills SR, Jackson DC, Older RA, Heaston DK, Moore AV. The incidence, etiologies, and avoidance of complications of pulmonary angiography in a large series. Radiology 1980;136:295-299.

21. Wicki J, Perneger TV, Junod AF, Bounameaux H, Perrier A. Assessing clinical probability of pulmonary embolism in the emergency ward: a simple score. Arch Intern Med 2001;161:92-97.

22. Qanadli SD, El Hajjam M, Mesurolle B, et al. Pulmonary embolism detection: prospective evaluation of dual-section helical CT versus selective pulmonary arteriography in 157 patients. Radiology 2000;217:447-455.

23. Raptopoulos V, Boiselle PM. Multi-detector row spiral CT pulmonary angiography: comparison with single-detector row spiral CT. Radiology 2001; 221:606-613.

24. Moreira JS, Alves MRA, da Silva LMC, et al. Trom- 
boembolismo pulmonar. In: da Silva LCC. Condutas em pneumologia. Rio de Janeiro: Revinter, 2001;693-703.

25. Moores LK, Jackson WL Jr, Shorr AF, Jackson JL. Meta-analysis: outcomes in patients with suspected pulmonary embolism managed with computed tomographic pulmonary angiography. Ann Intern Med 2004;141:866-874.

26. Patel S, Kazerooni EA, Cascade PN. Pulmonary embolism: optimization of small pulmonary artery visualization at multi-detector row CT. Radiology 2003;227:455-460.

27. Remy-Jardin M, Tillie-Leblond I, Szapiro D, et al. $\mathrm{CT}$ angiography of pulmonary embolism in patients with underlying respiratory disease: impact of multislice CT on image quality and negative predictive value. Eur Radiol 2002;12:1971-1978.

28. Tillie-Leblond I, Mastora I, Radenne F, et al. Risk of pulmonary embolism after a negative spiral CT angiogram in patients with pulmonary disease: 1year clinical follow-up study. Radiology 2002;223: 461-467.
29. Stein PD, Henry JW, Gottschalk A. Reassessment of pulmonary angiography for the diagnosis of pulmonary embolism: relation of interpreter agreement to the order of the involved pulmonary arterial branch. Radiology 1999;210:689-691.

30. Eyer BA, Goodman LR, Washington L. Clinicians "Response to Radiologists" reports of isolated subsegmental pulmonary embolism or inconclusive interpretation of pulmonary embolism using MDCT. AJR 2005;184:623-628.

31. Silva ISC, Muller NL. Diagnóstico por imagem do tromboembolismo pulmonar agudo. J Bras Pneumol 2004;30:474-479.

32. Lewis BD, James ME, Welch TJ, Joyce JW, Hallett JW, Weaver AL. Diagnosis of acute deep venous thrombosis of the lower extremities: prospective evaluation of color Doppler flow imaging versus venography. Radiology 1994;192:651-655.

33. Vaccaro JP, Cronan JJ, Dorfman GS. Outcome analysis of patients with normal compression US examinations. Radiology 1990;175:645-649.
34. Wells PS, Lensing AWA, Davidson BL, Prins MH, Hirsh J. Accuracy of ultrasound for the diagnosis of deep venous thrombosis in asymptomatic patients after orthopedic surgery: a meta-analysis. Ann Intern Med 1995;122:47-53.

35. Rollo HA, Maffei FHA, Lastória S, et al. Uso rotineiro da flebografia no diagnóstico da trombose venosa profunda dos membros inferiores. Cir Vasc Angiol 1986;2:7-12.

36. Coche EE, Hamoir XL, Hammer FD, Hainaut P, Goffette PP. Using dual-detector helical CT angiography to detect deep venous thrombosis in patients with suspicion of pulmonary embolism: diagnostic value and additional findings. AJR 2001;176: 1035-1039.

37. Loud PA, Katz DS, Klippenstein DL, Shah RD, Grossman ZD. Combined CT venography and pulmonary angiography in suspected thromboembolic disease: diagnostic accuracy for deep venous evaluation. AJR 2000;174:61-65. 